ITC $4 / 50$

Information Technology and Control

Vol. 50 / No. 4 / 2021

pp. $607-626$

DOI 10.5755/j01.itc.50.4.29690
Bayesian Processing of Data on Bursts of Pressure Vessels

\begin{tabular}{|l|l}
\hline Received 2021/08/30 & Accepted after revision 2021/11/05 \\
\hline
\end{tabular}

HOW TO CITE: Vaidogas, E. R. (2021). Bayesian Processing of Data on Bursts of Pressure Vessels. Information Technology and Control, 50(4), 607-626. https://doi.org/10.5755/j01.itc.50.4.29690

\title{
Bayesian Processing of Data on Bursts of Pressure Vessels
}

\section{Egidijus Rytas Vaidogas}

Vilnius Gediminas Technical University; Saulètekio al. 11, LT-10223, Vilnius, Lithuania; phone: +37068279620; e-mail: egidijus.vaidogas@vgtu.lt

Corresponding author: egidijus.vaidogas@vgtu.lt

Two alternative Bayesian approaches are proposed for the prediction of fragmentation of pressure vessels triggered off by accidental explosions (bursts) of these containment structures. It is shown how to carry out this prediction with post-mortem data on fragment numbers counted after past explosion accidents. Results of the prediction are estimates of probabilities of individual fragment numbers. These estimates are expressed by means of Bayesian prior or posterior distributions. It is demonstrated how to elicit the prior distributions from relatively scarce post-mortem data on vessel fragmentations. Specifically, it is suggested to develop priors with two Bayesian models known as compound Poisson-gamma and multinomial-Dirichlet probability distributions. The available data is used to specify non-informative prior for Poisson parameter that is subsequently transformed into priors of individual fragment number probabilities. Alternatively, the data is applied to a specification of Dirichlet concentration parameters. The latter priors directly express epistemic uncertainty in the fragment number probabilities. Example calculations presented in the study demonstrate that the suggested non-informative prior distributions are responsive to updates with scarce data on vessel explosions. It is shown that priors specified with Poisson-gamma and multinomial-Dirichlet models differ tangibly; however, this difference decreases with increasing amount of new data. For the sake of brevity and concreteness, the study was limited to fire induced vessel bursts known as boiling liquid expanding vapour explosions (BLEVEs).

KEYWORDS: post-mortem data, data scarcity, Bayesian updating, Poisson-gamma distribution, multinomial-Dirichlet distribution, epistemic uncertainty, aleatory uncertainty, explosion, pressure vessel, fragment, risk. 


\section{Introduction}

Bursts of pressure vessels occur as violent explosions often leading to a catastrophic fragmentation vessel shells. These explosions belong to the most violent industrial accidents [1]. The industry branch that is especially prone to pressure vessel explosions is related to handing of commercial hydrocarbons. Raw data on pressure vessel explosions is represented mainly by post-mortem information collected in the course of investigation of these accidents [9]. Nature of this data and its appropriateness to estimating consequences of future explosions is fairly diverse. However, a part of the data can be processed to the form suitable for a quantitative risk assessment (QRA) of facilities operating pressure vessels [33].

History of pressure vessel explosions in industry is long. However, the most violent explosions, like BLEVEs, are relatively rare events $[1,25]$. Amount of data on specific aspects of these events is not sufficient for a Fisherian (classical, frequentist) statistical analysis in the context of QRA. For instance, the largest widely known set of data on fragmentation of vessels due to BLEVEs consists of approximately 250 records and represents statistically highly nonhomogeneous population of these pressure containers [21, 38]. However, we think that limited data on vessel explosions is suitable for Bayesian analysis that forms the methodological basis of QRA [35].

The essential quantity characterising the immediate hazard of each vessel explosion is the number of fragments generated by vessel burst. This number determines to a great extent damaging effects of explosion. As a discrete random variable, the fragment number is susceptible of Bayesian estimation. The basic idea of this study was to carry out this estimation by applying two models of Bayesian reasoning known as compound Poisson-gamma (PG) and multinomial-Dirichlet (MD) distributions. They can be used for estimating probabilities of individual fragment numbers in terms of probability distributions expressing epistemic (reducible) uncertainty. Distributions of this type are widely used in the field of QRA [5].

The study presents a comparative analysis of PG and MD models with a view to applying them to QRA. Ultimate aim and, in our opinion, added value of the study was an improvement of QRA of pressure vessels through a better use of scarce data on catastrophic fragmentations of these containment tanks.

\section{Review of Related Work and Available Data}

The knowledge gap tackled in the present study is how to express uncertainty in the number of fragments by means of Bayesian reasoning. To date this uncertainty has been expressed simply by empirical proportions of individual fragment numbers counted for relatively small amount of explosion accidents (see the review in the book chapter [45] and references cited therein). These proportions can be easily supplemented with Fisherian confidence intervals, primarily with Clopper-Pearson intervals [7]. However, right here possibilities of the Fisherian estimation are exhausted. The precision of Fisherian confidence intervals will not increase significantly during a slow and sporadic arrival of new data on vessel fragmentations. In addition, it is difficult to propagate the confidence intervals through such QRA models as event trees and fault trees [36].

The current situation of data on fragment numbers is typical for most QRA applications that are predominantly based on Bayesian reasoning. Currently, there are three publicly accessible sources of information on numbers of fragments generated by BLEVEs:

1 In 1985, Holden and Reeves [14] presented counts of fragment numbers related to 23 fire induced vessel bursts. This original data has been directly gained from accident investigation reports. Indirect information presented in, say, scientific articles was not used.

2 In 2009, Gubinelli and Cozanni [13] declared a collection of data on fragmentation of 89 horizontal and vertical pressure vessels that underwent BLEVEs. Data has been extracted from original information presented by other authors. The same data was presented also by Tugnoli et al. [45].

3 In 2012, Sun et al. [38] presented counts of fragment numbers related to 259 BLEVEs of horizontal pressure vessels. The data has been acquired from books and scientific journals. This data was repeatedly presented by $\mathrm{Li}$ et al. [21]. The counts obtained by Sun et al. are given in Table 1 . They will be used for the example calculations presented in Section 6.

Counts of fragment numbers given in the sources just listed is processed data. These counts are not related 
to raw information on technical details of vessels, for instance, substance stored at the onset of failure, volume, wall thickness, relief pressure. Thus, the counts represent lumped, non-homogenous populations of vessels that underwent bursts. An attempt to divide these populations into more homogenous subpopulations will result in a decrease of the sets of events that underlie fragment number counts.

\section{Table 1}

Numbers of fragments counted after 259 BLEVE events [21,38]

\begin{tabular}{c|c|c} 
No. of fragments $n$ & Count $m_{n}$ & $\begin{array}{c}\text { Percentage } \\
m_{n} / n_{e} \times 100 \%^{\mathrm{a}}\end{array}$ \\
\hline 1 & 50 & 19.3 \\
\hline 2 & 98 & 37.8 \\
\hline 3 & 78 & 30.1 \\
\hline 4 & 24 & 9.27 \\
\hline 5 & 3 & 1.58 \\
\hline 6 & 2 & 0.77 \\
\hline 7 & 3 & 1.58 \\
\hline 8 & 0 & 0 \\
\hline 9 & 1 & 0.39 \\
\hline
\end{tabular}

${ }^{\mathrm{a}} n_{e}=259$; the subscript "e" means an explosion accident

Bayesian prediction of the number of fragments generated by pressure vessel bursts can be useful at least for three reasons:

1 Contrary to the Fisherian analysis, Bayesian reasoning allows to incorporate expert judgement into the estimation of the probabilities of individual fragment numbers. Clearly, the procedure used for this estimation must be sensitive to updates with scarce data on fragment numbers. In addition, this procedure should indicate an acceptable speed of learning from new data on vessel fragmentations [44].

2 Estimates of fragment number probabilities are expressed in the Bayesian format by prior and posterior distributions. They model epistemic uncertainty in these probabilities. Propagation of uncertainties expressed by Bayesian priors and posteriors through QRA models is fairly simple and transparent as compared with propagation of Fisherian confidence intervals [36].
3 The currently available methods of elicitation of the prior distributions related to PG and MD models allow to develop priors that express the analyst's attitude towards the situation of available knowledge on vessel fragmentation. This knowledge is relatively vague. From standpoint of simplicity and defensibility, diffuse non-informative priors are ideal [5].

A subjective estimation of an individual probability is a standard problem of Bayesian inference expressed as estimation of a single binomial parameter (binomial proportion) [17]. In the case under study, the observed data used for binomial modelling are number of vessel bursts with any number of fragments and number of cases with given number of fragments. Approaches to elicitation and updating of prior and posterior distributions of the binomial parameter used in the field of QRA are reviewed by Kelly and Smith [18] as well as Siu and Kelly [36]. A highly practical elicitation of a beta prior distribution for the binomial parameter used for QRA applications has been suggested by Atwood [4]. This distribution is called the constrained non-informative prior and it is suitable for updating with scarce data, among them data on vessel bursts.

The fact that a vessel burst can generate any number of fragments starting from one and this number is uncertain requires naturally to apply the multinomial generalisation of the binomial distribution as aleatory model of fragmentation. Bayesian inference in this case can be based on the compound MD distribution, in which probability vector (alpha-factors) drawn from a multi-parameter Dirichlet distribution will express probabilities of individual fragment numbers. MD distribution is the most commonly used model for common cause failures (CCFs) that are subject of QRA and reliability analysis [28, 44, 49]. Kelly and Atwood [16] suggested the so-called minimally informative Dirichlet prior distribution that is a generalisation of the constrained non-informative prior of the binomial parameter. This Dirichlet prior incorporates means of alpha-factors specified from data, if available, and is otherwise fairly diffuse.

Troffaes et al. [44] proposed to express epistemic uncertainty in the alpha-factors by means of lower and upper expectations of each alpha-factor as well as a learning parameter that determines how quickly the model learns from observed data. Elicitation of priors for alpha-factors is an extension of the minimally in- 
formative Dirichlet prior to the case where a precise mean for each alpha-factor cannot be specified.

The minimally informative Dirichlet prior will be used in this study for the estimation of fragment number probabilities. The first reason for this choice is that we have no zero counts related to the fragment numbers 1 to 4 that prevail in BLEVE events (Table 1). This allows a simple specification of means of marginal beta distributions used to construct the Dirichlet prior. The second reason is that an elicitation of minimally informative Dirichlet prior suggested by Kelly and Atwood [16] is simpler and more transparent than the specification of bounds of Dirichlet concentration parameters in line with the procedure proposed by Troffaes et al. [44].

Outside QRA and reliability analysis, the development of methods intended for eliciting priors of the MD model extends over fifty years since Lindley [22] proposed the combination of multinomial distribution and conjugate Dirichlet prior. Brief reviews of these methods are presented by Alvares et al. [2] as well as Elfadaly and Garthwaite [11]. Needless to say, a variety of prior elicitation methods and diversity of practical applications of MD model is large. Some of these methods are not suited to the data and modelling situation considered in this study as they deal, for instance, with elicitation of informative priors [47]. However, several methods developed outside QRA by Atwood [3], Bernard [6] and Walley [46] underlie directly or indirectly the aforementioned elicitation methods suggested for the case of CCFs. CCF events are similar to vessel fragmentations, because the number of explosions, $n_{e}$, can be treated as a total number of observed CCF events and the counts $m_{n}$ can be interpreted as counts of failure events with exactly $n$ failures (Table 1 ). This similarity was a substantial reason to use the aforementioned minimally informative Dirichlet prior distribution for the estimation of the fragment number probabilities in line with MD model.

An alternative approach to the Bayesian estimation of fragment number probabilities can be based on the fact that a slightly modified Poisson distribution is suitable, at least logically, for the description of integers representing fragment numbers. In the context of the present study, the usual Poisson data $\{r$ failures in $t$ time units\} should be interpreted as the total of $n_{+}$fragments in $n_{e}$ explosions $\}$. Probability masses of the Poisson distribution can be interpreted as probabilities of individual fragment numbers. The epistemic uncertainty is introduced into PG model as a prior of the Poisson parameter [17, 36].

The combination of aleatory Poisson model and epistemic gamma distribution has been used and is still used for a multitude of applications in various domains of science [10]. Applications of the PG model to QRA and reliability analysis are also numerous $[31,32]$. In the context of the present study, mention should be made of the attempt to expand modelling of uncertainties via PG model by introducing uncertainties in the Poison variables $r$ and $t$ (or $n_{+}$and $n_{e}$ in the case under study) $[26,27]$. The total number of fragments, $n_{+}$, can be inaccurate because of unreliable investigation of past explosion accidents and the number of explosions, $n_{e}$, can be uncertain, because not all explosions represented by the count $n_{e}$ may belong to the same category, say, fire induced BLEVEs.

A review of methods proposed for elicitation of prior for Poisson parameter in the context of QRA is presented by Kelly and Smith [17, 18]. The data situation related to vessel bursts and resulting fragment numbers suggests the constrained non-informative prior developed by Atwood for binomial modelling can also be used in case of PG model [4]. In the present study, the elicitation Poisson gamma prior was just an intermediate step in the estimation of fragment number probabilities with PG model. Our idea was to transform the epistemic uncertainty expressed by the gamma prior distribution into distributions of epistemic uncertainty related to Poisson probability masses. These epistemic distributions may serve as Bayesian estimates of the fragment number probabilities and make a viable alternative to the estimates expressed by the Dirichlet distribution. The application of PG model seems to be new, because attempts to model epistemic uncertainties in probability masses of Poisson and binomial distributions are not known to us.

In terms of safety engineering, the number of fragments is the essential information necessary for a probabilistic prediction of aerodynamic properties, trajectories and impact of fragments on vulnerable objects [34, 41, 45]. Eventually this information will be important to a design of protective structures intended for a defence against moderate-velocity and high-mass non-rigid projectiles from such events as BLEVEs. These structures are mainly safety barri- 
ers built in stationary facilities and along vulnerable roadside territory. In previous five decades, the design of safety barriers was mainly based on deterministic specification of blast and impact loads and deterministic assessment of structural response to these loads $[19,37,39,48]$. However, a probabilistic design of safety barriers is a more natural and scientifically justified approach due to considerable uncertainties on the side of blast and impact loads. To date attempts to design barriers in a probabilistic way were restricted mainly to the case of blast loading [15, 23, 29, 30, 40, 42, 43]. A probabilistic design of barriers for moderate-velocity impacts by non-totally rigid fragments from vessel bursts is in its infancy to the best of our knowledge. The Bayesian prediction of the number of fragments proposed in this study can be a useful, even if early, step towards non-deterministic assessment of impact loads and probabilistic design of safety barriers.

\section{Mathematical Problem Under Study}

The problem under study is expressed by a random number of fragments, $N$, generated per one vessel explosion. Formally, the number $N$ can be any integer greater than zero. However, real BLEVE events produce a very limited number of fragments. For instance, values of $N$ encountered in the past accidents range between 1 and 9 (Table 1 ).

The safety problem arises only when at least two fragments are ejected, that is, the random event $N \geq 2$ occurs (Figure 1). The event $N=1$ represents neg-

\section{Figure 1}

Illustration of the random events $N \geq 2$ : (a) ejection of two fragments (event $N=2$ ); (b) ejection of three or four fragments (events $N=3$ or $N=4$ )

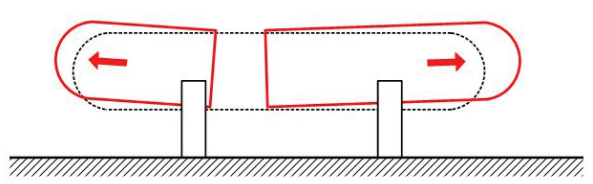

(a)

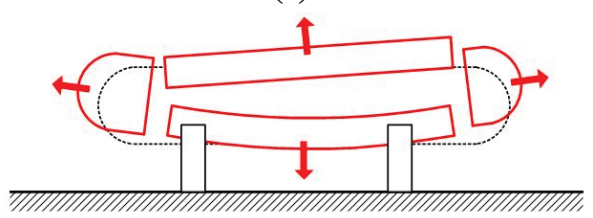

(b)

\section{Figure 2}

Illustration of the random events $N=1$ and $N \geq 5$ : (a) local crack is formed; (b) rupture without ejection of fragments; (c) generation of five or more small fragments

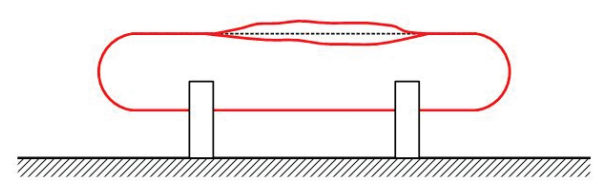

(a)

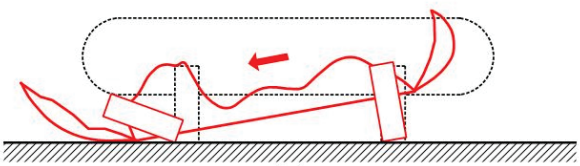

(b)

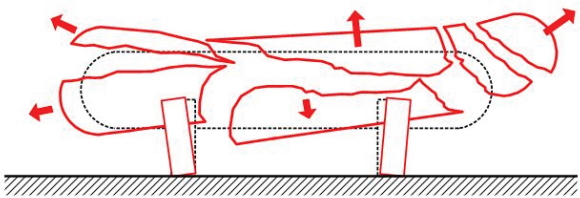

(c)

ligible hazard because it usually occurs as a vessel burst without long-range ejection of fragments (Figure 2ab). A generation of a large number of fragments represented by the event $N \geq 5$ poses relatively low risk. The kinetic energy of small and light fragments is comparatively low and they tend to have poor aerodynamic characteristics (Figure 2c). Due to the above reasons, it appears reasonable to restrict the prediction of two or more fragments. This leads to a consideration of 209 BLEVE events with proportions given in Table 2.

Explosion induced fragmentation of vessels is a very uncertain phenomenon. Therefore, the prediction of the number of fragments should have the form of the fragment number probabilities $P(N=n)$ with $n=1,2, \ldots$. They model aleatory (unreducible) uncertainty related to the random events $N=n$. Data on occurrences of the events $N=n$ is scarce and this results in fairly wide confidence intervals of $P(N=n)$. Clopper-Pearson confidence intervals of $P(N=n)$ calculated for the data on 209 BLEVE explosions are presented in Table 3.

Bayesian analysis yields estimates of the probabilities $P(N=n)$ expressed as prior and posterior probability distributions. In terms of QRA, prior and posterior related to $P(N=n)$ expresses the epistemic uncer- 


\section{Table 2}

Information extracted from Table 1 and related to the numbers of fragments recorded after 209 BLEVE events that generated at least two projectiles

\begin{tabular}{c|c|c|c}
\hline $\begin{array}{c}\text { No. of fragments } \\
n\end{array}$ & $\begin{array}{c}\text { Proportion } \\
m_{n} / n_{a}{ }^{\mathrm{a}}\end{array}$ & $\begin{array}{c}\text { Displaced } \\
\text { no. } n_{0}{ }^{\mathrm{b}}\end{array}$ & Category $k^{\mathrm{c}}$ \\
\hline 2 & 0.4689 & 0 & 1 \\
\hline 3 & 0.3732 & 1 & 2 \\
\hline 4 & 0.1148 & 2 & 3 \\
\hline 4 & 0.1148 & 2 & 3 \\
\hline 5 & 0.0144 & 3 & 4 \\
\hline 6 & 0.0096 & 4 & 5 \\
\hline 7 & 0.0144 & 5 & N/A \\
\hline 8 & 0 & 6 & N/A \\
\hline 9 & 0.0048 & 7 & N/A \\
\hline
\end{tabular}

${ }^{\mathrm{a}} m_{n}$ is the count of explosions with $n$ fragments, $n_{a}=209$

${ }^{\mathrm{b}} \mathrm{Cf}$ Figure 4 and Section 4.1

${ }^{\mathrm{c}}$ Cf Figure 4 and Section 5.1

\section{Table 3}

Clopper-Pearson $95 \%$ confidence intervals (CIs) obtained for the data pairs $m_{n}$ and $n_{a}(n=2,3,4)$

\begin{tabular}{c|c|c|c|c|c}
\hline$n$ & $m_{n}$ & $n_{a}$ & $\hat{\mu}_{n}$ & CI & Width of CI \\
\hline 2 & 98 & 209 & 0.4689 & $(0.400,0.5390)$ & 0.139 \\
\hline 3 & 78 & 209 & 0.3732 & $(0.307,0.443)$ & 0.136 \\
\hline 4 & 24 & 209 & 0.1148 & $(0.0750,0.1660)$ & 0.091 \\
\hline
\end{tabular}

tainty. In what follows, this uncertainty in $P(N=n)$ will be modelled by the random variables $\mathcal{P}_{n}$, the values of which will be denoted by $p_{n}$.

This study looks into the possibility to express uncertainties in the probabilities $P(N=n)$ by means of MG and PG models. They are adapted to the currently available empirical (post-mortem) data on numbers of fragments generated by BLEVEs and compared by applying to the same set of initial data.

The comparison is of the two models is based on two "common denominators":

1 The data used for eliciting priors for $P(N=n)$ is the same for both models. The data given in Table 2 was applied in the example calculation presented in Section 6.
2 Methods used for eliciting priors within PG and MD models are closely related and based on the same approach to Bayesian updating with scarce and slowly arriving data.

The sequence of reasoning based on the above "common denominators" is explained in Figure 3.

\section{Figure 3}

Processes used to predict fragment numbers by applying $\mathrm{PG}$ and MD models

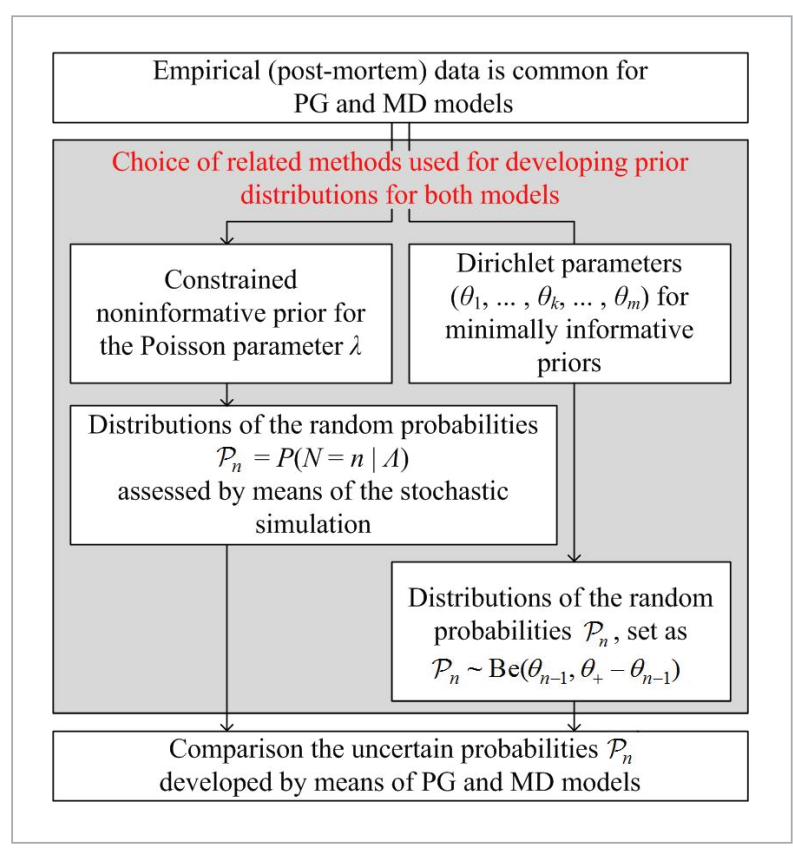

\section{Processing the Data by Means of the Poisson-Gamma Distribution}

\subsection{Distribution Adaptation the to the Case Under Study}

In context of the present study, the Poisson distribution is applied to modelling the number of fragments generated during one vessel explosion. The basic definition of the probability mass function of the Poisson distribution presumes that the number of occurrences takes on the values $0,1,2, \ldots$ and we are interested in values starting from two fragments, that is, from $n=2$. Therefore, the basic probability mass function should be applied to the displaced number of fragments, $N_{0}$, given by $N-2$. In this setting, the Poisson 
parameter $\lambda_{0}$ is equal to the expected value of $N_{0}$ and the probabilities $P(N=n)$ are equal to the probabilities $P\left(N_{0}=n-2\right)$. The probabilities $P(N=n)$ can be expressed through Poisson probability masses as follows:

$$
\begin{aligned}
P(N= & n)=P\left(N_{0}=n_{0} \mid \lambda_{0}\right)= \\
& =\frac{\lambda_{0}^{n_{0}}}{n_{0} !} \mathrm{e}^{-\lambda_{0}}, \quad n_{0}=0,1,2, \ldots .
\end{aligned}
$$

The numbers $n_{0}$ are illustrated in Table 2 and Figure 4. Let the random variable expressing uncertainty in the parameter $\lambda_{0}$ be $\Lambda_{0}$. In Bayesian analysis, the probability distribution of $\Lambda_{0}$ is called the prior or posterior for $\lambda_{0}$. The conjugate prior distribution of $\Lambda_{0}$ is the gamma distribution:

$\Lambda_{0} \sim \Gamma\left(\alpha_{0}, \beta_{0}\right)$,

where $\alpha_{0}$ is the shape parameter and $\beta_{0}$ is an inverse scale parameter. With the random parameter $\Lambda_{0}$, the uncertain probabilities $\mathcal{P}_{n}$ are expressed by

$$
\begin{aligned}
& \mathcal{P}_{n}=P\left(N_{0}=n_{0} \mid \Lambda_{0}\right)= \\
& =\frac{\Lambda_{0}^{n_{0}}}{n_{0} !} \mathrm{e}^{-\Lambda_{0}} \quad \text { with } n=n_{0}+2 .
\end{aligned}
$$

Consequently, uncertainty related to the fragment number probabilities $P(N=n)$ amounts in $\mathrm{PG}$ model to uncertainty related to a single Poisson parameter.

\section{Figure 4}

Illustration of the variables $n_{0}$ and $k$ used to describe fragment numbers in PG and MD analyses

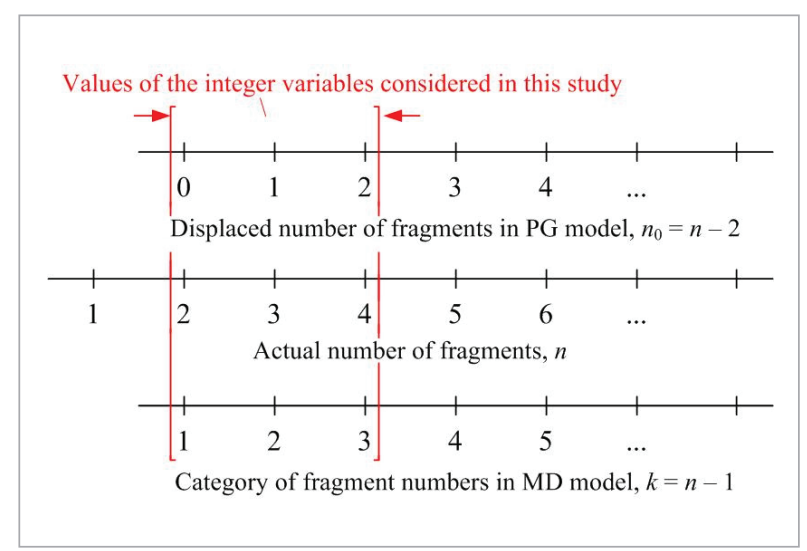

\subsection{Developing a Gamma Prior}

Developing the prior $\Gamma\left(\alpha_{0}, \beta_{0}\right)$ is the most problematic element of PG model. Naturally limited and sporadically incoming information on vessel explosions will require to develop a non-informative prior of $\Lambda_{0}$ that is relatively sensitive to updates with scarce new data. This problem is typical for QRA and can be addressed by eliciting the constrained non-informative prior distribution of $\Lambda_{0}$ [4]. It is suitable to incorporate limited empirical information on the average number of fragments and remains non-informative in general.

Two constraints are placed on the mean value of $N_{0}$ and the shape parameter $\alpha_{0}$, namely

$$
E\left[N_{0}\right]=\frac{\alpha_{0}}{\beta_{0}}=\hat{\lambda}_{0}
$$

$\alpha_{0}=05$,

where $\hat{\lambda}_{0}$ is the empirical average of the displaced number of fragments per explosion with at least two fragments. Thus the parameters of the prior distribution specified by Equations (4) are $\alpha_{0}=0.5$ and $\beta_{0}=0.5 / \hat{\lambda}$. As $\alpha_{0}<1$, the gamma prior $\Gamma\left(\alpha_{0}, \beta_{0}\right)$ has a J-shaped and monotonically decreasing probability density function. The coefficient of variation $(\mathrm{CoV})$ of $\Gamma\left(\alpha_{0}, \beta_{0}\right)$ is equal to $\alpha_{0}^{-1 / 2}$, that is, equal to $1.414(141 \%)$. Thus the assignment of the value 0.5 to the parameter $\alpha_{0}$ yields a large variance that corresponds to fairly large ignorance about the average number of fragments. The choice of this value is the only subjective element in the development of the constrained non-informative prior distribution.

In the present problem formulation, the new data will be the total displaced number of fragments, $n_{0+}$, counted in $n_{e}$ explosions:

$$
n_{0+}=\sum_{e=1}^{n_{e}}\left(n_{f e}-2\right),
$$

where $n_{f e}$ is the number of fragments generated by the explosion $e$. Then the posterior distribution based on the counts $n_{0+}$ and $n_{e}$ is $\Gamma\left(\alpha_{0}^{\prime}, \beta_{0}^{\prime}\right)$ with $\alpha_{0}^{\prime}=\alpha_{0}+n_{0+}$ and $\beta_{0}^{\prime}=\beta_{0}+n_{e}$. The count $n_{e}$ is the observed number of explosion accidents. Therefore the parameter $\beta_{0}$ can be viewed as a prior (pseudo) count of accidents. The parameter $\alpha_{0}$ can be interpreted as a prior pseudo-count of fragments in $\beta_{0}$ accidents. 
The relation between random probability $\mathcal{P}_{n}$ and random Poisson parameter $\Lambda_{0}$ given by Equation (3) is non-linear, and so the distribution of $\mathcal{P}_{n}$ can be estimated most easily by means of a stochastic (Monte Carlo) simulation. Results of such estimation are presented in Section 6. However, the distribution of $\mathcal{P}_{n}$ is not an end in itself from the viewpoint of QRA. Equation (3) can be used for generating values of $\mathcal{P}_{n}$ and simulating decisions concerning the number of fragments and related assessing of fragment characteristics [24].

\section{Processing the Data by Applying the Multinomial-Dirichlet Distribution}

\subsection{Distribution Adaptation to the Case Considered}

In the present context, the probability mass function of the multinomial distribution models an observation of $n_{e}$ explosions with $m$ categories of fragment numbers. Let the random number of observing the category $k$ in $n_{e}$ explosions be $M_{k}$. In the standard definition of the multinomial distribution, the number $k$ takes on values from 1 to $m$. However, we are interested in explosions generating two or more fragments. Therefore, the random variable $M_{k}$ must represent the number of occurrences of the random event $N=k+1$ (Figure 4). As $m$ is a fixed finite number, an application of the multinomial distribution must be preceded by the choice of the maximum limiting number of fragments $m+1$. The considerations presented in Section 3 suggest that $m+1$ can be equal to 4 or 5 . Then $m$ can be equal to 3 or 4 (Table 2 and Figure 4). Thus, in the present context, the multinomial probability mass function can be defined as

$$
\begin{aligned}
& P\left(\bigcap_{k=1}^{m}\left(M_{k}=m_{k}\right) \mid n_{e}, p_{1}, \ldots, p_{m}\right) \propto \prod_{k=1}^{m} p_{i}^{m} \text { with } \\
& \sum_{k=1}^{m} m_{k}=n_{e} \text { and } \sum_{k=1}^{m} p_{k}=1,
\end{aligned}
$$

where each $m_{k}$ is a non-negative integer and $p_{k}$ is the probability that a given explosion will generate $k+1$ fragments, that is, the probability

$$
p_{k}=P(N=k+1) \text {, }
$$

In terms of QRA, the probabilities $p_{k}$ are uncertain in the epistemic sense. The uncertainty in $p_{k}$ will be modelled by the random variables $\mathcal{P}_{k}$, the vector of which $\left(\mathcal{P}_{1}, \ldots, \mathcal{P}_{m}\right)$, follows a Dirichlet distribution:

$$
\left(\mathcal{P}_{1}, \ldots, \mathcal{P}_{k}, \ldots, \mathcal{P}_{m}\right) \sim \operatorname{Dir}\left(\theta_{1}, \ldots, \theta_{k}, \ldots, \theta_{m}\right)
$$

where $\theta_{k}$ are positive real numbers called the concentration parameters [16]. The parameters $\theta_{k}$ correspond to the uncertain probabilities $\mathcal{P}_{k}$. The marginal distributions of $\mathcal{P}_{k}$ are beta ones with parameters $\theta_{k}$ and $\theta_{+}-\theta_{k}$, where $\theta_{+}=\sum_{k=1}^{m} \theta_{k}$. That is, $\mathcal{P}_{k} \sim \operatorname{Be}\left(\alpha_{k}, \beta_{k}\right)$ with $\alpha_{k}=\theta_{k}$ and $\beta_{k}=\theta_{+}-\theta_{k}$. The marginal mean and variance of $\mathcal{P}_{k}$ are given by

$$
\begin{gathered}
E\left[\mathcal{P}_{k}\right]=\mu_{k}(\boldsymbol{\theta})=\frac{\theta_{k}}{\theta_{+}}, \\
V\left[\mathcal{P}_{k}\right]=\sigma_{k}^{2}(\boldsymbol{\theta})=\frac{\theta_{k}\left(\theta_{+}-\theta_{k}\right)}{\theta_{+}^{2}\left(\theta_{+}+1\right)} \frac{\theta_{k}}{\theta_{+}},
\end{gathered}
$$

where $\boldsymbol{\theta}$ denotes the vector $\left(\theta_{1}, \ldots, \theta_{k}, \ldots, \theta_{m}\right)$.

The Dirichlet distribution is a conjugate to the multinomial distribution. Thus the Dirichlet density

$$
\pi\left(p_{1}, \ldots, p_{m} \mid \theta_{1}, \ldots, \theta_{m}\right) \propto \prod_{k=1}^{m} p_{k}^{\theta_{k}-1}
$$

expresses a prior distribution of the probabilities $\mathcal{P}_{k}$.

The new data in the present context has the form $\left\{n_{f 1}, \ldots, n_{f k}, \ldots, n_{f m}\right\}$, where $n_{f k}$ is the number of fragmentations belonging to the category $k$ encountered in $n_{e}$ explosions. The number $n_{e}$ is equal to the sum $\sum_{e=1}^{n_{e}} n_{f e}$. The posterior distribution of $\mathcal{P}_{k}$ is again a Dirichlet with the updated concentration parameters $\theta_{k}^{\prime}=\theta_{k}+n_{f k}$. In other words, the count is $n_{f k}$ an observed number of vessel bursts into $k+1$ fragments.

As the count $n_{f k}$ is an observed number of explosion ns with $k+1$ fragments, the prior parameter $\theta_{k}$ can be treated as a prior pseudo-count of the $k+1$ explosions. Therefore, the larger is the sum of the pseudo-counts, $\theta_{+}$, the less diffuse is the prior distribution of the parameters $\theta_{k}$.

\subsection{Developing a Dirichlet Prior Distribution}

Developing a Dirichlet prior distribution consists in a selection of values for the parameters $\theta_{k}$. As the aim of this study is to compare PG and MD models, the 
Dirichlet prior can be developed by applying the same approach as in the case of the constrained non-informative gamma prior (Section 4.2). A minimally informative Dirichlet prior distribution can be elicited by means of the multivariate generalisation of the constrained non-informative prior method [16].

The following constraint is placed on the marginal means $E\left[\mathcal{P}_{k}\right]$ :

$$
E\left[\mathcal{P}_{k}\right]=\hat{\mu}_{k}=\frac{m_{k}}{n_{a}}
$$

where $m_{k}$ the observed number of accidents with $k+1$ fragments counted in $n_{a}$ explosion accidents. An example of $m_{k}, n_{a}$ and $\hat{\mu}_{k}$ is given in Table 2. The ratio $\hat{\mu}_{k}$ is the observed proportion and can be called the specified mean value. In principle, some values of $E\left[\mathcal{P}_{k}\right]$ can be specified subjectively if the proportions $\hat{\mu}_{k}$ are very small or equal to zero [16]. However, the data used in this study does not contain "empty" or "almost empty" categories $k$ related to the small numbers of fragments posing the most serious impact hazard (Table 2).

Parameters of the marginal prior, $\alpha_{k}$ and $\beta_{k}$, are specified by means of the following expressions:

$$
\alpha_{k}=0.5 \text { and } \beta_{k}=\frac{0.5\left(1-\hat{\mu}_{k}\right)}{\hat{\mu}_{k}} \text { if } \hat{\mu}_{k}<0.5
$$

and

$$
\alpha_{k}=\frac{0.5 \hat{\mu}_{k}}{1-\hat{\mu}_{k}} \text { and } \beta_{k}=0.5 \text { if } \hat{\mu}_{k}>0.5 \text {. }
$$

The parameters $\alpha_{k}$ and $\beta_{k}$ calculated by means of Equations (11) allow to specify a marginal variance of of $E\left[\mathcal{P}_{k}\right]$ related to the specified mean value $\hat{\mu}_{k}$ :

$$
\hat{\sigma}_{k}^{2}=\frac{\hat{\mu}_{k}\left(1-\hat{\mu}_{k}\right)}{\alpha_{k}+\beta_{k}+1} .
$$

With the parameters $\alpha_{k}$ and $\beta_{k}$, parameters of the Dirichlet prior, $\theta_{k}$, are calculated by a constraint minimisation of the least squares objective function:

$$
\varphi(\boldsymbol{\theta})=\left(\sum_{k=1}^{m}\left(\hat{\mu}_{k}-\mu_{k}(\boldsymbol{\theta})\right)^{2}+\left(\hat{\sigma}_{k}^{2}-\sigma_{k}^{2}(\boldsymbol{\theta})\right)^{2}\right)
$$

with the constraint that each $\theta_{k}>0$. The functions $\mu_{k}(\boldsymbol{\theta})$ and $\sigma_{k}^{2}(\boldsymbol{\theta})$ in the above minimisation problem are expressed by Equations (9). Reasons for the minimization of $\varphi(\boldsymbol{\theta})$ were explained by Kelly and Atwood [16].

The initial point of the minimisation of $\varphi(\theta)$ can be the vector $\boldsymbol{\theta}$ with the components calculated by means of the Equations (12), that is, $\theta_{k}=\alpha_{k}$.

With the desired parameters $\theta_{k}$, the epistemic probability distributions of the fragment number probabilities will be

$\mathcal{P}_{n} \sim \operatorname{Be}\left(\theta_{n-1}, \theta_{+}-\theta_{n-1}\right)$

or

$\mathcal{P}_{n} \sim \operatorname{Be}\left(\theta_{n-1}^{\prime}, \theta_{+}^{\prime}-\theta_{n-1}^{\prime}\right)$

depending on the prior or posterior status of the concentration parameters. In Equations (14), the quantity $\theta_{+}^{\prime}$ is the sum of the updated parameters $\theta_{k}^{\prime}$.

The above specification of the epistemic uncertainty distributions of $\mathcal{P}_{n}$ is based only on the Dirichlet distribution. An application of the epistemic distributions of fragment number probabilities to purposes of QRA will require sampling values of $\mathcal{P}_{n}$ form a Dirichlet distribution. It is commonly known that this can be done with relative ease by sampling from the gamma distributions $\Gamma\left(\theta_{n-1}, 1\right)$ [12].

\section{A Numerical Example}

The starting point of this case study is data on fragment numbers counted in 209 BLEVE accidents and given in Table 2. This data will be used for both PG and MD modelling (Figure 3). The proportions given in Table 2 and denoted by $\hat{\mu}_{n}$ suggest that the prevailing numbers of fragments are 2, 3 and 4 . Therefore, prior and posterior distributions of the random probabilities $\mathcal{P}_{n}$ will be developed only for $n=2,3$ and 4. This means that the indices shown in Figure 4 will take on the following values: $n_{0}=0,1,2$ and $k=1,2$, 3 . The case of five fragments $(n=5)$ was not included in the analysis due to its small observed proportion $\hat{\mu}_{5}=0.0144$. The dealing with only three fragment numbers 2, 3 and 4 allowed a visualisation of Dirichlet results by means of ternary graphs. 


\subsection{Application of the Poisson-gamma Model}

From Table 2, the average displaced number of fragments per accident is

$$
\hat{\lambda}_{0}=\sum_{n_{0}=0}^{7} n_{0} \hat{\mu}_{n_{0}}=0 \times 0.4689+\ldots+7 \times 0.0432=0.790 \text {. }
$$

Pairs of proportions $\hat{\mu}_{n_{0}}$ and Poisson probability masses $P\left(N_{0}=n_{0} \mid 0.790\right)\left(n_{0}=0,1, \ldots, 7\right)$ are compared in Table 3 . The difference between $\hat{\mu}_{n_{0}}$ and $P\left(N_{0}=n_{0} \mid 0.790\right)$ is relatively low, and so we assumed that the goodness of fit of Poisson distribution is sufficient for the purposes of this case study.

\section{Table 3}

Comparison of proportions $\hat{\mu}_{n_{0}}$ and Poisson probability masses calculated with $\hat{\lambda}_{0}=0.790$

\begin{tabular}{c|c|c|c}
\hline$n$ & $n_{0}$ & $\hat{\mu}_{n_{0}}$ & $P\left(N_{0}=n_{0} \mid 0.790\right)$ \\
\hline 2 & 0 & 0.4689 & 0.4538 \\
\hline 3 & 1 & 0.3732 & 0.3585 \\
\hline 4 & 2 & 0.1148 & 0.1416 \\
\hline 5 & 3 & 0.0144 & 0.0373 \\
\hline 6 & 4 & 0.0096 & 0.0074 \\
\hline 7 & 5 & 0.0144 & 0.0012 \\
\hline 8 & 6 & 0 & $0.015 \times 10^{-2}$ \\
\hline 9 & 7 & 0.0048 & $0.0017 \times 10^{-2}$ \\
\hline
\end{tabular}

The parameters of the prior distribution of $\Lambda_{0}$ specified by Equations (4) are $\alpha_{0}=0.5$ and $\beta_{0}=0.5 / \hat{\lambda}=$ $0.5 / 0.79=0.633$. Assume that in four future explosions with $n \geq 2$ the following fragment numbers $n_{f e}$ will be counted: $\{4,2,3,3\}$. From Equation (5), $n_{0+}=4$. Thus the new data is $\left\{n_{0+}, n_{e}\right\}=\{4,4\}$. Applying $\left\{n_{0+}, n_{e}\right\}$ yields the posterior parameters: $\alpha_{0}^{\prime}=\alpha_{0}+$ $n_{0 t}=0.5+4=4.5$ and $\beta_{0}^{\prime}=\beta_{0}+n_{e}=0.633+4=4.633$. Thus the posterior distribution of $\Lambda_{0}$ is $\Gamma(4.5,4.633)$. Densities of $\Gamma(0.5,0.633)$ and $\Gamma(4.5,4.633)$ are plotted in Figure 5. Characteristics of $\Gamma(0.5,0.633)$ and $\Gamma(4.5,4.633)$ are given in Table 4.

Prior and posterior distributions of the uncertain probabilities $\mathcal{P}_{n}$ were assessed by means of a stochastic simulation. A total of 10000 simulation runs were

\section{Figure 5}

Prior and posterior densities obtained for the mean $\lambda_{0}$ of the random displaced number of fragments, $N_{0}$

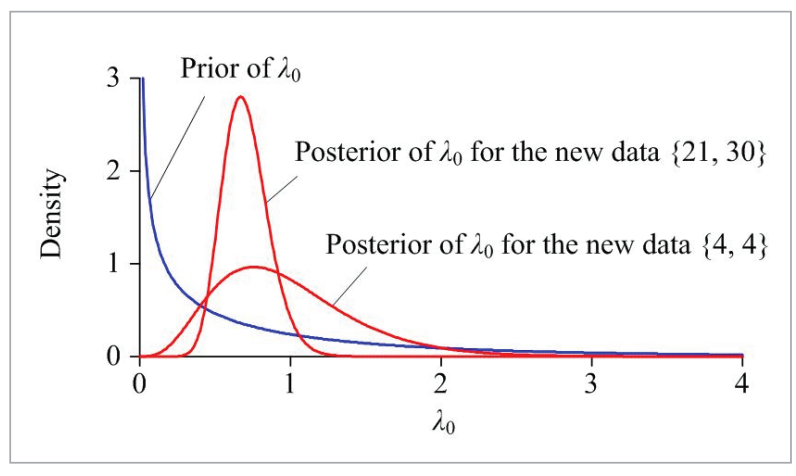

Table 4

Properties of gamma priors and posteriors of $\Lambda_{0}$

\begin{tabular}{l|c|c}
\hline \multicolumn{1}{c|}{ Property } & $\begin{array}{c}\text { Prior } \\
\Gamma(0.5,0.633)\end{array}$ & $\begin{array}{c}\text { Posterior } \\
\Gamma(4.5,4.633)\end{array}$ \\
\hline Mean & 0.790 & 0.971 \\
\hline CoV & $141 \%$ & $47.1 \%$ \\
\hline Quantile $\lambda_{0,0.05}$ & 0.003 & 0.359 \\
\hline Median $\lambda_{0,0.5}$ & 0.359 & 0.900 \\
\hline Quantile $\lambda_{0,0.95}$ & 3.03 & 1.826 \\
\hline
\end{tabular}

carried out using the transformation given by Equation (3). The simulation generated two groups of samples consisting of prior and posterior values of $\mathcal{P}_{n}$ :

$$
\begin{aligned}
& \boldsymbol{p}_{n}=\left\{\frac{\lambda_{0 i}^{n_{0}}}{n_{0} !} \mathrm{e}^{-\lambda_{0 i}}, i=1 \ldots, M\right\}(n=1,2,3), \\
& \boldsymbol{p}_{n}^{\prime}=\left\{\frac{\lambda_{0 i}^{n_{0}}}{n_{0} !} \mathrm{e}^{-\lambda_{0 i}^{\prime}}, i=1 \ldots, M\right\}(n=1,2,3),
\end{aligned}
$$

where $\lambda_{0 i}$ and $\lambda_{0 i}^{\prime}$ are values of $\Lambda_{0}$ generated in the simulation run $i$ from $\Gamma(0.5,0.633)$ and $\Gamma(4.5,4.633)$, respectively; $M$ is the number of simulation runs. Descriptive measures of the samples $\boldsymbol{p}_{n}$ and $\boldsymbol{p}_{n}^{\prime}$ are presented in Table 5. Frequency distributions (polygons) of $\boldsymbol{p}_{n}$ and $\boldsymbol{p}_{n}^{\prime}$ are plotted in Figure 6. The limitation of arguments of the prior and posterior distri- 
Table 5

Characteristics of the samples $\boldsymbol{p}_{n}$ and $\boldsymbol{p}_{n}^{\prime}$ used to assess the distributions of the random variables $\mathcal{P}_{n}$

\begin{tabular}{c|c|c|c|c|c|c}
\hline Sample & Mean & CoV, $\%$ & $5^{\text {th }}$ perc. & $95^{\text {th }}$ perc. & Min & Max \\
\hline $\boldsymbol{p}_{2}$ & 0.626 & 51.0 & 0.051 & 0.997 & 0.000 & 1.000 \\
\hline $\boldsymbol{p}_{3}$ & 0.190 & 68.0 & 0.003 & 0.366 & 0.000 & 0.368 \\
\hline $\boldsymbol{p}_{4}$ & 0.086 & 110 & 0.000 & 0.265 & 0.000 & 0.271 \\
\hline $\boldsymbol{p}_{2}^{\prime}$ & 0.417 & 39.1 & 0.162 & 0.700 & 0.034 & 0.944 \\
\hline $\boldsymbol{p}_{3}^{\prime}$ & 0.331 & 13.8 & 0.234 & 0.368 & 0.056 & 0.368 \\
\hline $\boldsymbol{p}_{4}^{\prime}$ & 0.162 & 42.8 & 0.045 & 0.266 & 0.002 & 0.271 \\
\hline
\end{tabular}

butions of $\mathcal{P}_{3}$ and $\mathcal{P}_{4}$ by the intervals $[0,37]$ and $[0,28]$ is explained in Appendix A.

An additional comment is necessary if we look at values of the simulated probabilities given by

$$
\begin{aligned}
& p_{n i}=\lambda_{0 i}^{n_{0}} \mathrm{e}^{-\lambda_{0 i}} / n_{0} !\left(n_{0}=n-2 ; n=2,3,4\right), \\
& p_{n i}^{\prime}=\lambda_{0 i}^{n_{0}} \mathrm{e}^{-\lambda_{0 i}^{\prime}} / n_{0} !\left(n_{0}=n-2 ; n=2,3,4\right) .
\end{aligned}
$$

The values $p_{n i}$ and $p_{n i}^{\prime}$ simulate probability masses of the Poisson distribution and are components of the samples $\boldsymbol{p}_{n}$ and $\boldsymbol{p}_{n}^{\prime}$, respectively. In each simulation run $i, p_{n i}$ and $p_{n i}^{\prime}$ were computed by generating three values of $\lambda_{0 i}$ and three values of $\lambda_{0 i}^{\prime}$. Each of them was sampled independently from respective prior and posterior gamma distributions. This led to the result that the totals

$$
p_{+, i}=\sum_{n=2}^{4} p_{n i} \text { and } p_{+, i}^{\prime}=\sum_{n=2}^{4} p_{n i}^{\prime}
$$

do not come to some constant value as would be the case with the fixed Poisson probability masses $P\left(N_{0}=n_{0} \mid \lambda_{0}\right) \quad\left(n_{0}=0,1,2\right)$. In addition, values of $p_{+, i}$ and $p_{+, i}^{\prime}$ in some simulation runs exceeded unity (Figure 7). However, proportions of the probabilities $p_{n i}$ and $p_{n i}^{\prime}$ in the sums $p_{+, i}$ and $p_{+, i}^{\prime}$ can be utilised for QRA. They allow to sample values of the fragment number probabilities as explained in Appendix B.

\section{Figure 6}

Estimates of priors and posteriors of the probabilities $P(N=n)$ obtained with PG model: (a) the case $N=2$; (b) the case $N=3$; (c) the case $N=4$

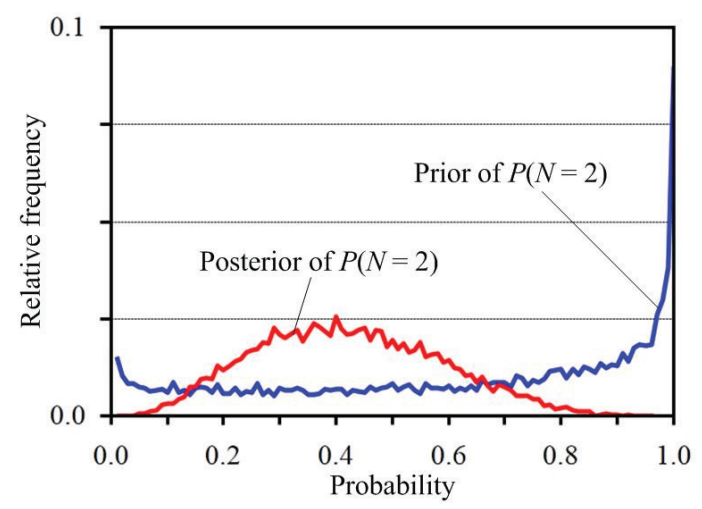

(a)

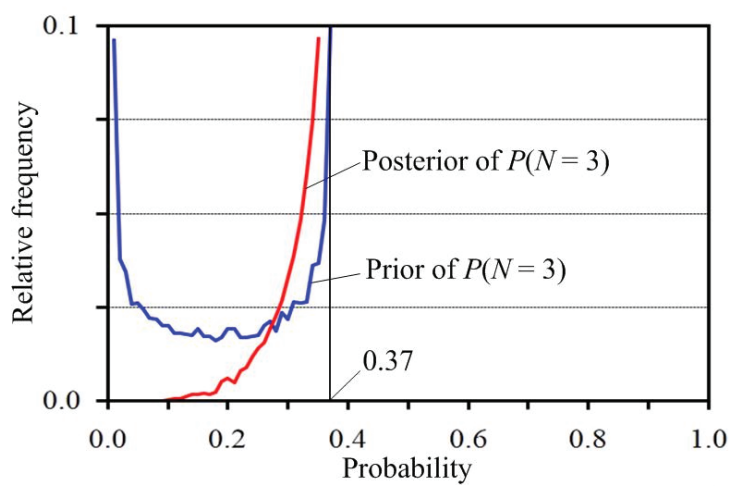

(b)

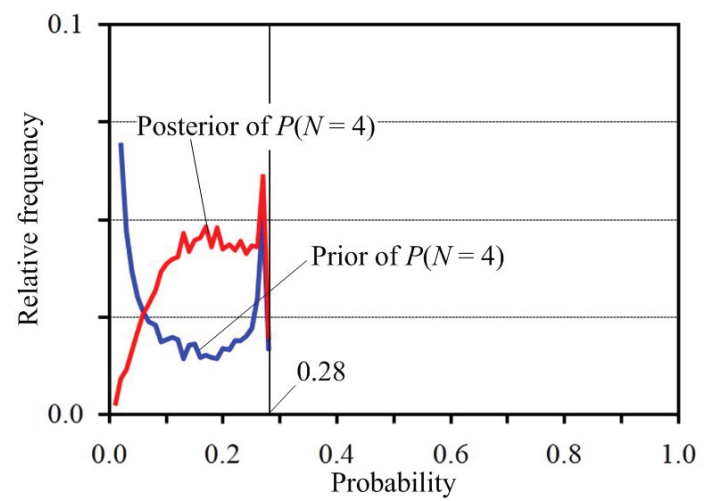

(c) 


\section{Figure 7}

Distributions of the simulated sums $p_{+, i}$ and $p_{+, i}^{\prime}$ computed by means of Equations (18)

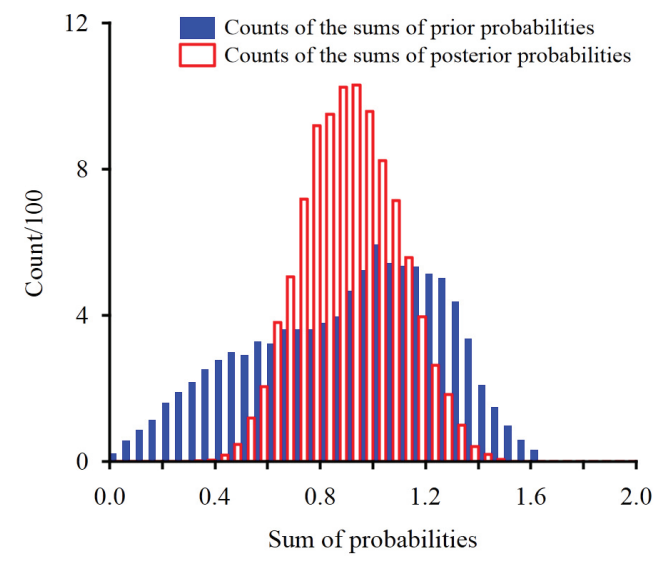

\subsection{Application of the Multinomial-Dirichlet Model}

In case of MD model, prior distributions of the random probabilities $\mathcal{P}_{n}(n=2,3,4)$ were developed by applying the observed proportions $\hat{\mu}_{k}(k=n-1)$ given in Table 2. Values of $\hat{\mu}_{k}$ were used to specify the marginal means $E\left[\mathcal{P}_{k}\right]$ of $\mathcal{P}_{k}$ according to Equation (11).

The parameters of the marginal beta priors, $\alpha_{k}$ and $\beta_{k}$, were calculated by means of Equations (12). Values of $\alpha_{k}$ and $\beta_{k}$ as well as values of the marginal variances $\hat{\sigma}_{k}^{2}$ calculated by means of Equation (13) are given in Table 6.

\section{Table 6}

The specified marginal prior beta distributions $\operatorname{Be}\left(\alpha_{k}, \beta_{k}\right)$ calculated with Equations (12) and (13)

\begin{tabular}{c|c|c|c|c|c}
\hline$n$ & $k$ & $\hat{\mu}_{k}$ & $\alpha_{k}$ & $\beta_{k}$ & $\hat{\sigma}_{k}^{2}$ \\
\hline 2 & 1 & 0.4689 & 0.5 & 0.5663 & 0.1205 \\
\hline 3 & 2 & 0.3732 & 0.5 & 0.8398 & 0.1000 \\
\hline 4 & 3 & 0.1148 & 0.5 & 3.8554 & 0.0190 \\
\hline
\end{tabular}

The values $\alpha_{k}$ were grouped into the vector $\theta=$ $(0.5,0.5,0.5,0.5)$. This vector was taken as the initial point of the minimisation of the function $\varphi(\theta)$ defined by Equation (14). A ternary graph of $\varphi(\theta)$ is shown in Figure 8. The minimum of $\varphi(\theta)$ was found at the point $\boldsymbol{\theta}^{*}=\left(\theta_{1}^{*}, \theta_{2}^{*}, \theta_{3}^{*}\right)=(0.66,0.53,0.17)$. The function value $\varphi\left(\theta^{*}\right)$ is equal to 0.00161 . The sum of the minimising values $\theta_{k}^{*}$ is $\theta_{+}=1.36$. The prior marginal distributions of $\mathcal{P}_{n}$ are the beta distributions $\operatorname{Be}(0.66,1.36), \operatorname{Be}(0.53,1.36)$ and $\operatorname{Be}(0.17,1.36)$ with densities plotted in Figure 9. Properties of these distributions are given in Table 7 . A ternary graph of the prior Dirichlet density $\pi\left(p_{2}, p_{3}, p_{4} \mid 0.66,0.53,0.17\right)$ is presented in Figure 10.

\section{Figure 8}

Ternary graph of the least squares function $\varphi(\boldsymbol{\theta})$ defined by Equation (14)

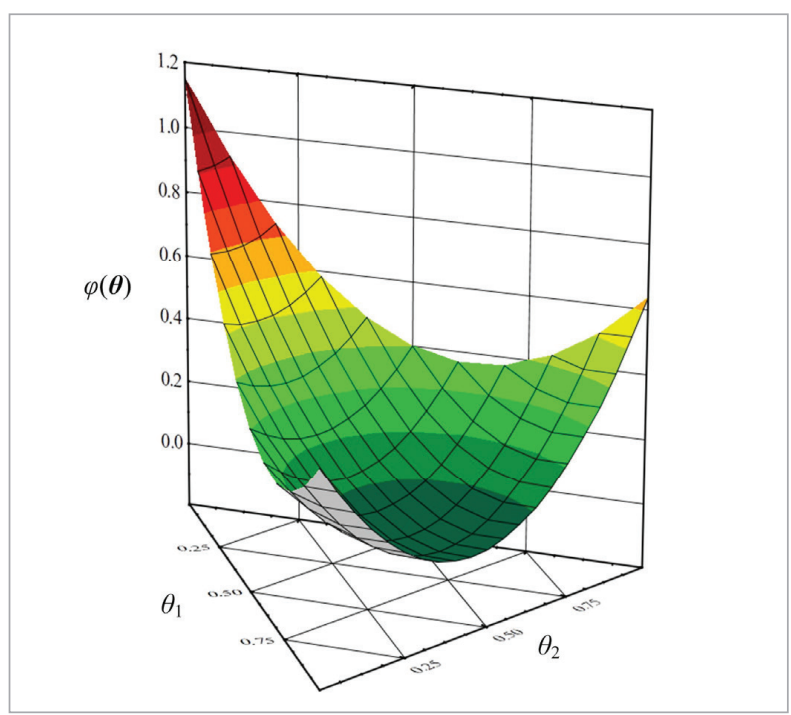

\section{Figure 9}

Prior and posterior beta densities of the fragment number probabilities $P(N=n)(n=2,3,4)$

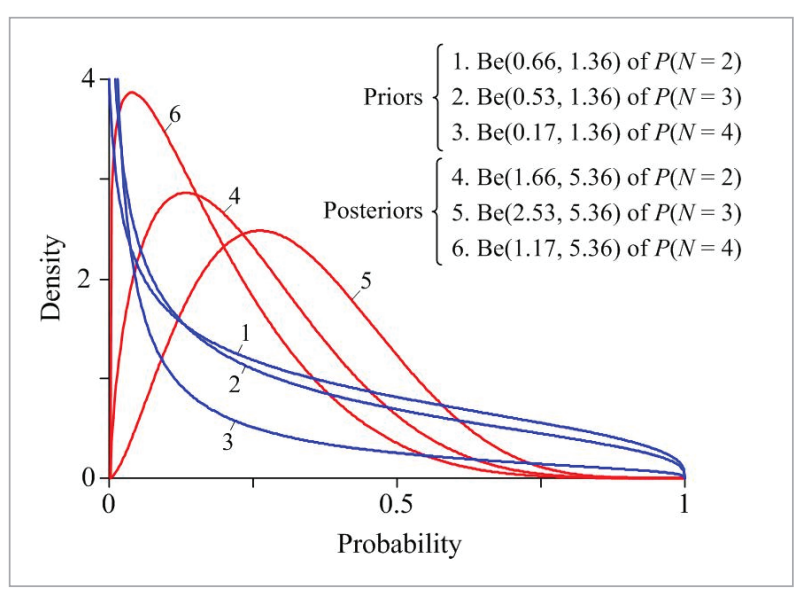


Table 7

Properties of the marginal prior and posterior beta distributions of the random probabilities $\mathcal{P}_{n}(n=2,3,4)$

\begin{tabular}{l|c|c}
\hline \multicolumn{1}{c|}{ Property } & $\begin{array}{c}\text { Prior beta densi- } \\
\text { ties with } \theta_{+}=1.36\end{array}$ & $\begin{array}{c}\text { Posterior beta densi- } \\
\text { ties with } \theta_{+}^{\prime}=5.36\end{array}$ \\
\hline Mean of $\mathcal{P}_{2}$ & 0.3267 & 0.2365 \\
\hline CoV of $\mathcal{P}_{2}$ & $82.6 \%$ & $63.5 \%$ \\
\hline 5th perc. of $\mathcal{P}_{2}$ & 0.00757 & 0.0394 \\
\hline 95th perc. of $\mathcal{P}_{2}$ & 0.8448 & 0.5225 \\
\hline Mean of $\mathcal{P}_{3}$ & 0.2804 & 0.3207 \\
\hline CoV of $\mathcal{P}_{3}$ & $94.2 \%$ & $48.8 \%$ \\
\hline 5th perc. of $\mathcal{P}_{3}$ & 0.00244 & 0.0920 \\
\hline 95th perc. of $\mathcal{P}_{3}$ & 0.8157 & 0.6037 \\
\hline Mean of $\mathcal{P}_{4}$ & 0.1111 & 0.1792 \\
\hline CoV of $\mathcal{P}_{4}$ & $178 \%$ & $78.0 \%$ \\
\hline 5th perc. of $\mathcal{P}_{4}$ & $7.51 \times 10^{-8}$ & 0.0157 \\
\hline 95th perc. of $\mathcal{P}_{4}$ & 0.5940 & 0.4567 \\
\hline
\end{tabular}

Let new information acquired for updating the Dirichlet prior $\pi\left(p_{2}, p_{3}, p_{4} \mid 0.66,0.53,0.17\right)$ be same as in $P G$ analysis, namely, $\{4,2,3,3\}$ (four explosions with 4, 2, 3 and 3 fragments). Then the new data has the form $\left\{n_{f 1}, n_{f 2}, n_{f 3}\right\}=\{1,2,1\}$. With the new data, the vector of posterior Dirichlet parameters, $\left(\theta_{1}^{\prime}, \theta_{2}^{\prime}, \theta_{3}^{\prime}\right)$, is $(1.66,2.53,1.17)$ and the posterior sum $\theta_{+}^{\prime}$ is equal to 5.36. Figure 11 presents a ternary graph of the $\mathrm{Di}$ richlet posterior $\pi\left(p_{2}, p_{3}, p_{4} \mid 1.66,2.53,1.17\right)$. The surfaces shown in Figures 10 and 11 are cubic approximations of the Dirichlet densities fitted to precise values of the Dirichlet prior $\operatorname{Dir}(0,66,0,53,0,17)$ and posterior $\operatorname{Dir}(1,66,2,53,1,17)$. This explains the negative density values that appear in Figure 11. However, the approximate surfaces are sufficient to illustrate the degree of the change resulting from the Bayesian updating of the Dirichlet prior with new data.

\subsection{Comparison of the Models Under Study}

The next natural step of this case study is a comparison of results obtained by means of PG model and the model based on the specification of Dirichlet parameters. As declared in Figure 3, either of them was applied to the same data presented in Table 2 and related methods were used to develop non-informative
Figure 10

Ternary graph of the Dirichlet prior $\pi\left(p_{2}, p_{3}, p_{4} \mid 0.66,0.53,0.17\right)$

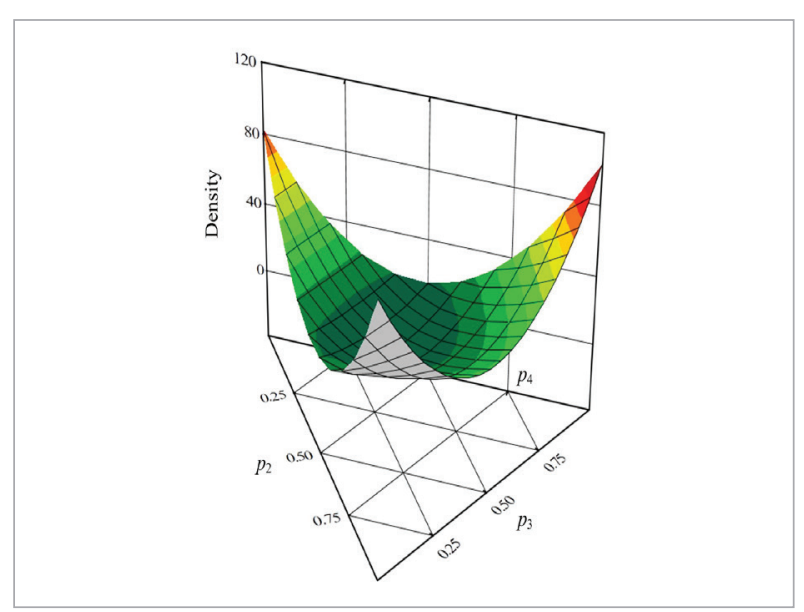

Figure 11

Ternary graph of the Dirichlet posterior $\pi\left(p_{2}, p_{3}, p_{4} \mid 1.66,2.53,1.17\right)$

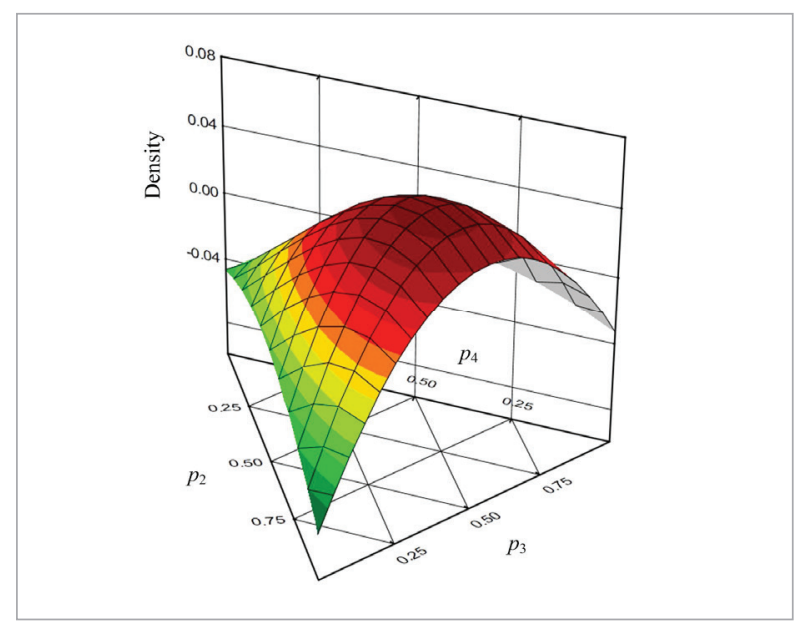

priors for these models. Both models yield estimates of the fragment number probabilities $P(N=n)$ expressed as distributions of the epistemic random variables $\mathcal{P}_{n}(n=2,3,4)$. Yet there the similarity ends. The comparison must be made between distributions of $\mathcal{P}_{n}$ independently assessed by means a stochastic simulation and multivariate distribution of the triplet of random variables $\left(\mathcal{P}_{2}, \mathcal{P}_{3}, \mathcal{P}_{4}\right)$.

The shape of the epistemic distributions of $P(N=n)$ developed by means of PG model is determined by the function of Poisson probability masses (see Figure 6 and Appendix A). The positive, practical feature of this model is that the estimation of the probabilities 
$P(N=n)$ requires eliciting only one prior distribution, namely, the distribution of the average number of accidents per explosion. This quantity has a clear physical meaning and is well understandable for the analyst. The application of the Poisson distribution is also natural due to the range of values of $N$ and incidence of fragment numbers.

The distributions of $P(N=n)$ specified by means of PG model have all three distinctive forms ("bell", "J" and "U" shapes). These forms are determined by the choice of gamma prior and nature of the Poisson model. In other words, estimates of $P(N=n)$ are as good as is the prior distribution of the displaced average number of fragments, $\lambda_{0}$. The procedure suggested for developing the constrained non-informative gamma prior of $\lambda_{0}$ is in essence formal and allows little leeway for subjective judgement. However, the mere decision to apply this procedure is subjective.

The shape of the marginal beta priors and posteriors developed within MD model is a direct choice dictated by this model. Figures 5 and 10 as well as Tables 5 and 7 reveal that these marginal beta priors differ significantly from the priors developed by simulation within PG model. Another difference between these two models lies in the nature of the quantities, for which priors must be developed. MD model requires to specify priors for probabilities. Thus, the analyst will have to do with quantifying the degree of belief instead of quantifying uncertainty in the average number of fragments. The latter task is obviously more natural than the former.

The suggested procedure used for developing minimally informative prior distribution of the multinomial parameters is also technically formal. It relieves the analyst of making decisions concerning the probabilities $P(N=n)$ that could be overly subjective. This procedure is a multivariate generalisation of the constrained non-informative procedure used in PG model. Thus, the degree of subjectivity in the use of the minimally informative priors is minimal. However, the choice of the minimally informative procedure is subjective in principle.

It seems that the only practical approach allowing to compare PG model with MD model is looking at convergence of Bayesian estimates of $P(N=n)$ with larger amounts of data. Hypothetical future data was used to demonstrate convergence of this type in QRA applications [36]. A widely known review of BLEVE accidents from the years 1926 to 2004 reveals that accidents recorded on the global scale happen roughly 1 to 4 times per year [1]. Thus, formally in the coming decade we can expect 10 to 40 BLEVE accidents and the past data reveals that approximately $80 \%$ of them will generate at least two fragments [38]. Thus, the potential amount of new information that could be gained from the future accidents and used for Bayesian updating may be extracted from 8 to 32 accidents.

Let us assume that in the coming ten years we will face 30 accidents with fire induced vessel disintegrations in at least two fragments (30 realisations of the random events $N \geq 2$ ). As the period of one decade will hardly produce essential changes in design and exploitation of pressure vessels, it is likely that proportions of fragment numbers will be similar to the values of $\hat{\mu}_{n}$ given in Table 2 . Therefore, hypothetical, future data may have the form given in Table 8 .

\section{Table 8}

Hypothetical data on thirty future 30 BLEVE accidents $\left(n_{e}=30\right)$

\begin{tabular}{c|c|c|c|c}
\hline$n$ & $m_{n}$ & $\hat{\mu}_{n}=\frac{m_{n}}{n_{e}}$ & $n_{0}$ & $k$ \\
\hline 2 & 14 & 0.467 & 0 & 1 \\
\hline 3 & 11 & 0.367 & 1 & 2 \\
\hline 4 & 5 & 0.167 & 2 & 3 \\
\hline
\end{tabular}

The total number of fragments counted in these 30 accidents is equal to 81. In line with Equation (5), the corresponding sum of displaced numbers of fragments, $n_{0+}$, is equal to $81-2 \times n_{e}$, that is, 21 . The new data for updating the gamma prior of the uncertain Poisson parameter $\Lambda_{0}$ has the form $\left\{n_{0+}, n_{e}\right\}$ and amounts to $\{21,30\}$. With the new data, the posterior gamma parameters will be $\alpha_{0}^{\prime}=\alpha_{0}+n_{0+}=0.5+$ $21=21.5$ and $\beta_{0}^{\prime}=\beta_{0}+n_{e}=0.633+30=30.6$. The density of the posterior distribution $\tilde{\mathrm{A}}(21.5,30.6)$ is shown in Figure 5. The mean, CoV and $90 \%$ credible interval of $\tilde{A}(21.5,30.6)$ are equal to $0.703,21.6 \%$ and $(0.473,0.969)$, respectively. The credible interval is illustrated in Appendix A.

The transformation of the uncertainty expressed by the posterior distribution $\tilde{\mathrm{A}}(21.5,30.6)$ yielded three samples $\boldsymbol{p}_{n}^{\prime}(n=2,3,4)$ of $\mathcal{P}_{n}$ values. Descriptive sta- 
Table 9

Characteristics of the samples $\boldsymbol{p}_{n}^{\prime}$ used to express uncertainty in the probabilities $\mathcal{P}_{n}$ and related to ten years data on future BLEVE accidents

\begin{tabular}{c|c|c|c|c|c|c}
\hline Sample & Mean & CoV, \% & $\begin{array}{c}5^{\text {th }} \\
\text { perc. }\end{array}$ & $\begin{array}{c}95^{\text {th }} \\
\text { perc. }\end{array}$ & Min & Max \\
\hline $\boldsymbol{p}_{2}^{\prime}$ & 0.500 & 14.76 & 0.378 & 0.622 & 0.250 & 0.760 \\
\hline $\boldsymbol{p}_{3}^{\prime}$ & 0.341 & 6.81 & 0.295 & 0.367 & 0.209 & 0.368 \\
\hline $\boldsymbol{p}_{4}^{\prime}$ & 0.122 & 27.01 & 0.070 & 0.179 & 0.029 & 0.240 \\
\hline
\end{tabular}

tistics and frequency polygons of $\boldsymbol{p}_{n}^{\prime}$ are given in Table 9 and Figure 12, respectively.

In the case of the Dirichlet model, the hypothetical new information is expressed by the counts related to the fragment number categories $k$, namely, $\left\{n_{f 1}, n_{f 2}, n_{f 3}\right\}=\{14,11,5\}$ (see Table 8). Adding these counts to the prior values of the Dirichlet parameters, $(0.66,0.53,0.17)$, yields the posterior parameters $\left(\theta_{1}^{\prime}, \theta_{2}^{\prime}, \theta_{3}^{\prime}\right)=(14.7,11.5,5.17)$ with the sum $\theta_{+}^{\prime}=31.4$. Densities of the marginal posterior distributions $\mathrm{Be}(14.7,31.4), \mathrm{Be}(11.5,31.4)$ and $\mathrm{Be}(5.17,31.4)$ are shown in Figure 12. Properties of these distributions are listed in Table 10.

Table 10

Marginal posterior beta distributions calculated with the hypothetical ten years data given (cf Table 8)

\begin{tabular}{l|c|c|c}
\hline Probability & Distribution & Mean & CoV, \% \\
\hline$P(N=2)$ & $\operatorname{Be}(14.7,31.4)$ & 0.314 & 21.3 \\
\hline$P(N=3)$ & $\operatorname{Be}(11.5,31.4)$ & 0.268 & 24.9 \\
\hline$P(N=4)$ & $\operatorname{Be}(5.17,31.4)$ & 0.141 & 40.2 \\
\hline
\end{tabular}

As expected, the relatively large amount of data presented in Table 8 resulted in peaked (comparatively narrow) posterior distributions of $\mathcal{P}_{n}$. The distributions developed by PG and MD models came closer together. However, a particularly close convergence was not achieved. In average, estimates of the probabilities $P(N=2)$ and $P(N=3)$ produced by the hypothetical 10 years data were tangibly larger in the

\section{Figure 12}

Three pairs of posteriors of the probabilities $P(N=n)$ $(n=2,3,4)$ calculated for supposed new data by means of $\mathrm{PG}$ and MD models

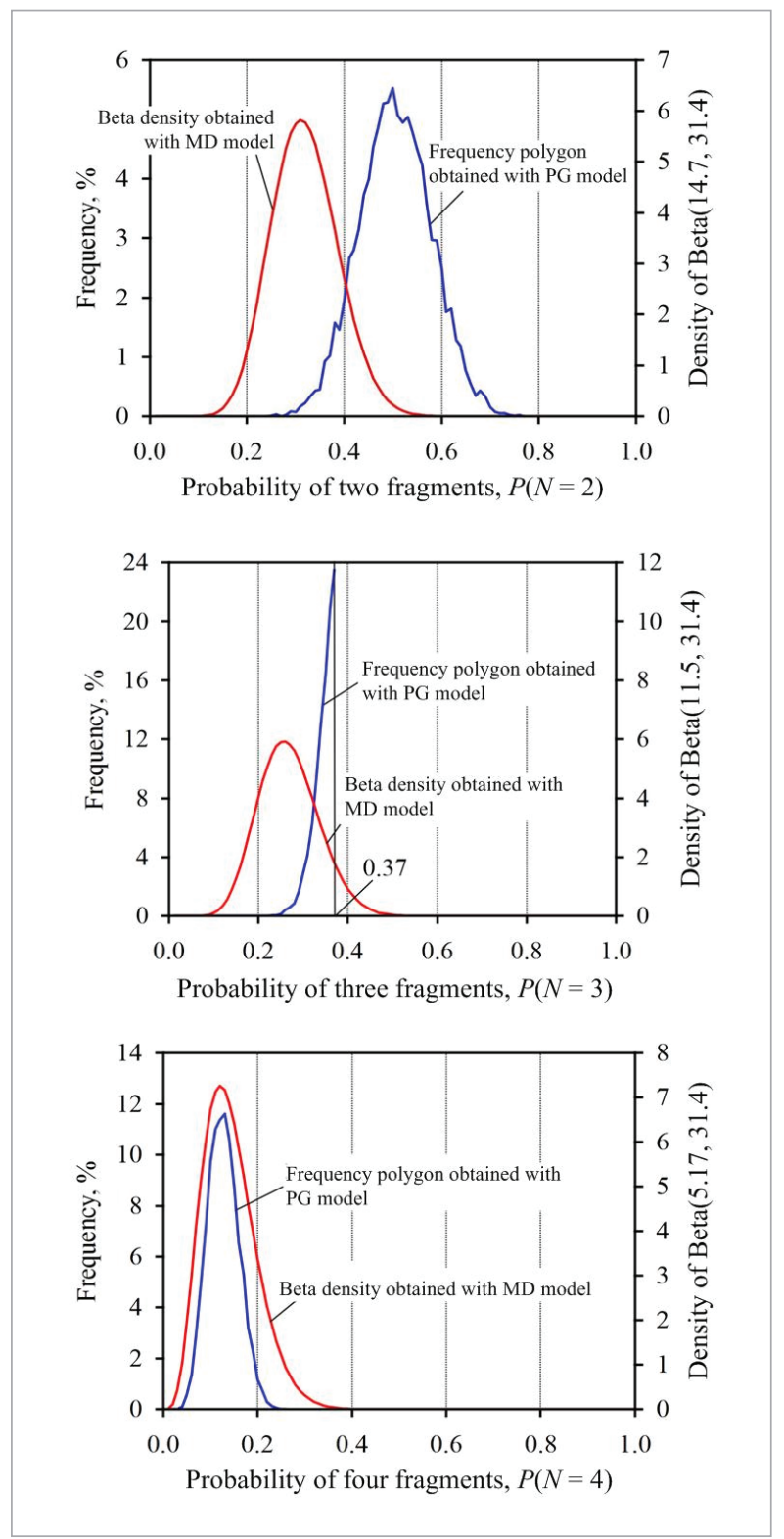

case of PG model (cf Tables 9 and 10). It is very likely that convergence of results obtained with these models will increase with a further increase of the amount of data. However, the speculation about the data that will become available in the coming two or three decades is of little practical use. 


\section{Discussion}

An answer to the question, which of the two models, PG or MD, should be used for QRA applications, is not straightforward. In the present data situation, either of the models yields fairly vague prior estimates of the fragment number probabilities (Figures 6 and 9 ). These estimates are non-informative prior distributions that express diffuse statements about the probabilities $P(N=n)$. Thus we have to compare two diffuse priors without knowing true values of the probabilities $P(N=n)$. In addition, an estimation of these probabilities is only an early, if not the first, step of QRA related to pressure vessel explosions. Uncertainties related to the probabilities $P(N=n)$ must be propagated further and transformed eventually into uncertainties in fragment impact probabilities.

It seems that the only worthwhile answer to the above question can be provided from the heuristic and conservative point of view:

1 The three fragmentation events $N=n(n=2,3,4)$ must be ranked in terms of the hazard of fragment impacts on potential targets. A stochastic simulation of fragment ejection and projection can be used for this ranking [34, 41].

2 The most hazardous fragmentation must be associated with the prior (posterior) distribution of the model that yields larger values of the probability of fragment number in question.

For instance, the vessel fragmentation into two oblong end-caps shown in Figure 1a might be found as the most hazardous event due to largest kinetic energy of these fragments at the instant of ejection. Then the prior (posterior) distribution developed by means of PG model must be preferred. This distribution covers larger range of values of the probability $P(N=2)$ than the prior (posterior) elicited by means of MD model (compare the prior and posterior percentile ranges $(0.051,0.997)$ and $(0.162,0.700)$ given in Tables 5 and to the corresponding ranges $(0.00757,08448)$ and (0.0394, 0.5225) shown in Table 7).

In the context of the present modelling, the aleatory parts of PG or MD models were used only formally, as platforms for eliciting priors of fragment number probabilities. The aleatory Poisson distribution served as means for propagating epistemic uncertainty in average number of fragments per accident and the aleatory multinomial distribution was only background for the application of the Dirichlet prior and posterior distributions. The fact that Poisson and multinomial distributions were not directly used for modelling allows to relax the assumption of constant parameters of these distributions. In the presence of time trends, Bayesian inference for these parameters will be more complicated but still tractable $[8,17,20]$. Here, it is pertinent to note that to date applications of MD model to CCFs have not included checks for trends in occurrences of these failures $[28,44,49]$.

The processed data on fragment numbers presented in Section 2 does not allow to reveal the trend of these numbers over previous time represented by a series of BLEVE explosions. However, this data is suitable for eliciting non-informative priors in line with $\mathrm{PG}$ or $\mathrm{MD}$ models. Elicitation of non-informative priors is not a rigorous procedure and includes a good deal of subjectivity. A part of this subjectivity can be disregarding of a possible time trend in average number of fragments.

\section{Conclusion}

Two Bayesian approaches to a prediction of the number of fragments from pressure vessel explosions have been proposed. The prediction was expressed in terms of probabilities of individual fragment numbers. The proposed approaches were based on an application of two Bayesian models known as compound Poisson-gamma (PG) and multinomial-Dirichlet (MD) probability distributions.

The study reached the conclusion that either of the compound distributions can be used to elicit prior distributions of fragment number probabilities on the basis of available post-mortem data on fragmentation of pressure vessels. Updating these epistemic distributions with the new data consists in updating priors of Poisson lambda parameter in PG model and Dirichlet concentration parameters in MD model.

The priors of the fragment number probabilities developed by means of PG and MD models for currently available scarce data on vessel fragmentations differ noticeably. However, these priors are only the first step of the Bayesian updating procedure. Calculations with hypothetical new data brought out that difference between posterior distributions obtained with PG and MD models tend to decrease with increasing 
amount of new data. Another conclusion that can be drawn from the example calculations presented in the study is that priors expressing epistemic uncertainty in fragment number probabilities are sensitive to updating with scarce new data. This conclusion is highly advantageous because such vessel explosions as BLEVEs are rare events even on the global scale. Thus the arrival of new data on these accidents is slow.

Findings of this study can be useful for a quantitative assessment of risk posed by potential damage due to fragment impacts. The priors and posteriors of the uncertain fragment number probabilities can be propagated with relative ease through the logical model of QRA known as the event tree. This model is naturally suited to express scenarios of the accident involving a pressure vessel burst.

\section{Appendix A}

The probability mass function of the Poisson distribution is used to express uncertainty in the fragment number probabilities $P(N=n)$ expressed by the probability masses $P\left(N_{0}=n-2\right)$ (see Equation (1)). Epistemic uncertainty related to the probabilities $P\left(N_{0}=n-2 \mid \lambda_{0}\right)$ is expressed by propagating uncertainty in the Poisson parameter $\lambda_{0}$ through the function $\lambda_{0}^{n_{0}} \mathrm{e}^{-\lambda_{0}} / n_{0}$ ! with $n_{0}=n-2$. This function takes the forms $\mathrm{e}^{-\lambda_{0}}, \lambda_{0} \mathrm{e}^{-\lambda_{0}}$ and $0.5 \lambda_{0}^{2} \mathrm{e}^{-\lambda_{0}}$ for the values 0,1 and 2 of the displaced number of fragments $n_{0}$. Graphs of these functions are given in Figure A. We can see that in the case of the functions $P\left(N_{0}=1 \mid \lambda_{0}\right)$ and $P\left(N_{0}=2 \mid \lambda_{0}\right)$ probability values are limited by the intervals $[0,0.368]$ and [0, 0.271], respectively. This limitation stems from the structure of the Poisson distribution and explains limits of the prior and posterior densities of $P(N=3)$ and $P(N=4)$ shown in Figures $6 \mathrm{~b}$ and $6 \mathrm{c}$.

\section{Appendix B}

The triplets $\left(p_{2 i}, p_{3 i}, p_{4 i}\right)$ or $\left(p_{2 i}^{\prime}, p_{3 i}^{\prime}, p_{4 i}^{\prime}\right)$ calculated with Equations (18) are suitable for generating fragment numbers in individual runs of a stochastic simulation aimed at predicting vessel fragmentation effects. A number of fragments in the run $i$ can be generated by sampling a value $u_{i}$ of a random variable $U$ that is uniformly distributed over the interval ] $0, p_{+, i}[$ or $] 0, p_{+, i}^{\prime}$ [ (Figure B).

\section{Figure A}

Graphs of the Poisson probability masses expressed as functions of the Poisson parameter $\lambda_{0}$ : (a) graph of $\mathrm{e}^{-\lambda_{0}}$, (b) graph of $\lambda_{0} \mathrm{e}^{-\lambda_{0}}$, (c) graph of $0.5 \lambda_{0}^{2} \mathrm{e}^{-\lambda_{0}}$

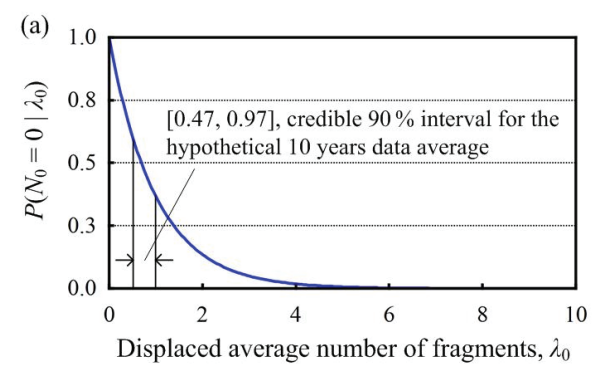

(a)

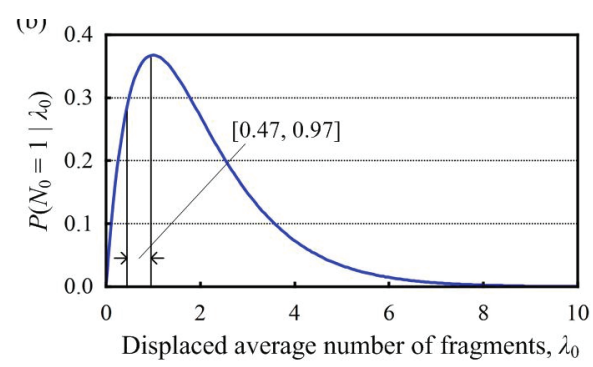

(b)

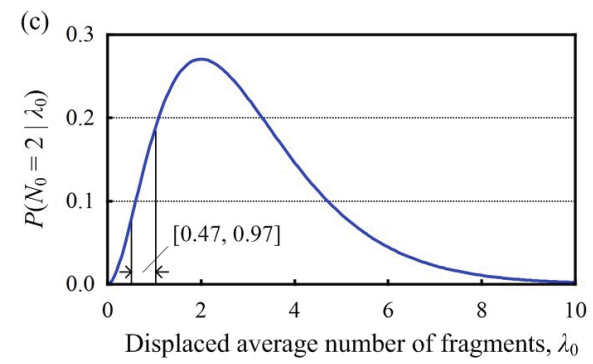

(c)

\section{Figure B}

Two approaches to generating the number of fragments in a stochastic simulation run $i$ : (a) ejection of three fragments is simulated when $p_{+, i}<1$; (b) ejection of four fragments is simulated when $p_{+, i}>1$

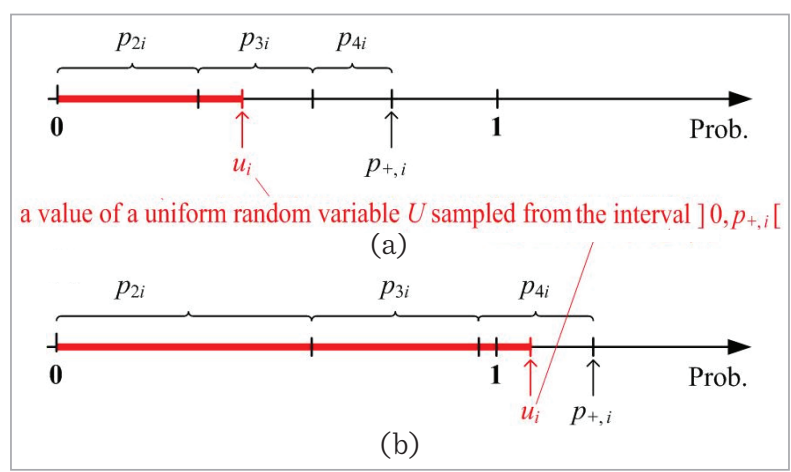




\section{References}

1. Abbasi, T., Abbasi, S. A. The Boiling Liquid Expanding Vapour Explosion (BLEVE): Mechanism, Consequence Assessment, Management. Journal of Hazardous Materials, 2007, 141(3), 489-519. https://doi.org/10.1016/j. jhazmat.2006.09.056

2. Alvares, D., Armero, C., Forte, A. What does objective mean in a Dirichlet-Multinomial Process? International Statistical Review, 2017, 86(1), 106-118. https://doi. org/10.1111/insr.12231

3. Atwood, C. L. Consequences of Mapping Data or Parameters in Bayesian Common-Cause Analysis. Reliability Engineering \& System Safety, 2013, 118(C), 118-131. https://doi.org/10.1016/j.ress.2013.04.015

4. Atwood, C. L. Constrained Noninformative Priors in Risk Assessment. Reliability Engineering \& System Safety, 1996, 53(1), 37-46. https://doi.org/10.1016/09518320(96)00026-9

5. Bandyopadhyay, P. S., Brittan, G., Taper, M. L. Belief, Evidence, and Uncertainty: Problems of Epistemic Inference. Springer, Berlin, 2016. https://doi. org/10.1007/978-3-319-2777r2-1

6. Bernard, J. M. An Introduction to the Imprecise Dirichlet Model for Multinomial Data. International Journal of Approximate Reasoning, 2005, 39(2-3), 123-150. https://doi.org/10.1016/j.ijar.2004.10.002

7. Brown, L. D., Tony Cai, T., Das Gupta, A. Interval Estimation for a Binomial Proportion. Statistical Science, 2001, 16(2), 101-133. https://doi.org/10.1214/ ss/1009213286

8. Cargnoni, C., Muller, P., West, M. Bayesian Forecasting of Multinomial Time Series Through Conditional Gaussian Dynamic Models. Journal of the American Statistical Association, 1997, 92(438), 640-647. https:// doi.org/10.2307/2965711

9. CCPS. Guidelines for Investigating Chemical Process Incidents, 2nd ed. Center of Chemical Process Safety, New York, 2003.

10. Congdon, P. D. Applied Bayesian Hierarchical Methods. CRC Press, Boca Raton.

11. Elfadaly, F. G., Garthwaite, P. H. Eliciting Dirichlet and Gaussian Copula Prior Distributions for Multinomial Models. Statistical Computation, 2017, 27, 449-467. https://doi.org/10.1007//s11222-016-9632-7

12. Frigyik, B. A., Kapila, A., Gupta, M. R. Introduction to the Dirichlet Distribution and Related Processes. Tech- nical Report UWEETR-2010-006. University of Washington, Washington, 2010.

13. Gubinelli, G., Cozzani, V. Assessment of Missile Hazards: Identification of Reference Fragmentation Patterns. Journal of Hazardous Materials, 2009, 163, 10081018. https://doi.org/10.1016/j.jhazmat.2008.07.056

14. Holden, P. L., Reeves, A. B. Fragment Hazards from Failures of Pressurized Liquefied Gas Vessels. IChemE Symposium Series, 1985, 93, 205-217.

15. Juocevicius, V., Vaidogas, E. R. Effect of Explosive Loading on Mechanical Properties of Concrete and Reinforcing Steel: Towards Developing a Predictive Model. Mechanika, 2010, 81(1), 5-12.

16. Kelly, D., Atwood, C. L. Finding a Minimally Informative Dirichlet Prior Distribution Using Least Squares. Reliability Engineering \& System Safety, 2011, 96(3), 398402. https://doi.org/10.1016/j.ress.2010.11.008

17. Kelly, D., Smith, C. Bayesian Inference for Probabilistic Risk Assessment: A Practitioner's Guidebook. Springer, London, 2011. https://doi.org/10.100r/978-1-84996-187-5

18. Kelly, D., Smith, C. Bayesian Inference in Probabilistic Risk Assessment - The Current State of the Art. Reliability Engineering and System Safety, 2009, 94(2), 628-643. https://doi.org/10.1016/j.ress.2008.07.002

19. Lam, N., Yong, A. C. Y., Lam, C., Kwan, J., Perera, J., Disfani, M., Gad, E. Displacement-based Approach for the Assessment of Overturning Stability of Rectangular Rigid Barriers Subjected to Point Impact. Journal of Engineering Mechanics, 2018, 144(2), 04017161-1 04017161-15. https://doi.org/10.1061/(ASCE)EM.19437889.0001383

20. Lee, Y. J. Test of Trend in Count Data: Multinomial Distribution Case. Journal of the American Statistical Association, 1980, 75(372), 1010-1014. https://doi.org/10.1 080/01621459.1980.10477588

21. Li, Z., Sun, D., Sun, J., Jiang, J. Study on the Number of Primary and Secondary Fragments Produced by Explosion of Horizontal Vessel. Lecture Notes in Civil Engineering, 2021, 110, 399-40\%. https://doi. org/10.1007/978-981-15-9121-1_28

22. Lindley, D. The Bayesian Analysis of Contingency Tables. The Annals of Mathematical Statistics, 1964, 35(4), 1622-1643. https://doi.org/10.1214/aoms/1177700386

23. Linkute, L., Juocevicius, V., Vaidogas, E. R. A Probabilistic Design of Sacrificial Cladding for a Blast Wall Us- 
ing Limited Statistical Information on Blast Loading. Mechanika, 2013, 19(1), 58-66. https://doi.org/10.5755/ j01.mech.19.1.3621

24. Mannan, S. (Ed.). Lees's Loss Prevention in the Process Industries, 4th ed. Elsevier, Amsterdam, 2012.

25. Mannan S. (Ed.). Lees's Process Safety Essentials. Hazard Identification, Assessment and Control. Elsevier, Amsterdam, 2014. https://doi.org/10.1016/B978-185617-776-4.00006-3

26. Martz, H. F., Hamada, M. S. Uncertainty in Counts and Operating Time in Estimating Poisson Occurrence Rates. Reliability Engineering \& System Safety, 2003, 80(1), 75-79. https://doi.org/10.1016/S09518320(02)00267-3

27. Martz, H. F., Picard, R. R. Uncertainty in Poisson Event Counts and Exposure Time in Rate Estimation. Reliability Engineering \& System Safety, 1995, 48(3), 181190. https://doi.org/10.1016/0951-8320(95)00019-X

28. Mi, J., Beer, M., Li, Y.-F., Broggi, M., Cheng, Y. Reliability and Importance Analysis of Uncertain System with Common Cause Failures Based on Survival Signature. Reliability Engineering \& System Safety, 2020, 201, 106988. https://doi.org/10.1016/j.ress.2020.106988 https://doi.org/10.1016/j.ress.2020.106988

29. Nagata, M., Beppu, M., Ichino, H., Yashiro, H. Proposal on Risk Assessment of Reinforced Concrete Structures Subjected to Explosive Loads. International Journal of Protective Structures, 2017, 8(3), 407-432. https://doi. org/10.1177/2041419617721549

30. Netherton, M. D., Stewart, M. G. Risk-Based Blast-Load Modelling: Techniques, Models and Benefits. International Journal of Protective Structures, 2016, 7(3), 430451. https://doi.org/10.1177/2041419616666455

31. Nguyen, H. D., Gouno, E. Bayesian Inference for Common Cause Failure Rate Based on Causal Inference with Missing Data. Reliability Engineering \& System Safety, 2020, 197, 106789. https://doi.org/10.1016/j. ress.2019.106789

32. Nguyen, H. D., Gouno, E. Maximum Likelihood and Bayesian Inference for Common-Cause of Failure Model. Reliability Engineering \& System Safety, 2019, 182, 2019(February), 56-62, https://doi.org/10.1016/j. ress.2018.10.003

33. Nolan, D. Handbook of Fire and Explosion Protection Engineering Principles for the Oil, Gas, Chemical, and Related Facilities, 4nd ed. Elsevier, Amsterdam, 2019.

34. Pula, R., Khan, F. I., Veitch, B., Amyotte, P. R. A Model for Estimating the Probability of Missile Impact: Mis- siles Originating from Bursting Horizontal Cylindrical Vessels. Process Safety Progress, 2006, 26(2), 129-139. https://doi.org/10.1002/prs.10178

35. Singpurwalla, N. D. Reliability and Risk: A Bayesian Perspective. Wiley, New York, 2006. https://doi. org/10.1002/9780470060346

36. Siu, N. O., Kelly, D. L. Bayesian Parameter Estimation in Probabilistic Risk Assessment. Reliability Engineering \& System Safety, 1998, 62(1-2), 89-116. https://doi. org/10.1016/S0951-8320(97)00159-2

37. Smith. P. D. Blast Walls for Structural Protection Against High Explosive Threats: A Review. International Journal of Protective Structures, 1(1), 67-84. https:// doi.org/10.1260/2041-4196.1.1.67

38. Sun, D., Jiang, J., Zhang, M., Wang, Z., Huang, G., Qiao, J. Parametric Approach of the Domino Effect for Structural Fragments. Journal of Loss Prevention in the Process Industries, 2012, 25(1), 114-126. https://doi. org/10.1016/j.jlp.2011.06.029

39. Sun, J., Lam, N., Zhang, L., Ruan, D., Gad, E. Computer Simulation of Contact Forces Generated by Impact. International Journal of Structural Stability and Dynamics, 2017, 17 (1), 1750005-1750015. https://doi. org/10.1142/S0219455417500055

40. Vaidogas, E. R. On Applying Sparse and Uncertain Information to Estimating the Probability of Failure due to Rare Abnormal Situations. Information Technology and Control, 2009, 38(2), 135-146.

41. Vaidogas, E. R. Predicting the Ejection Velocities of Fragments from Explosions Cylindrical Pressure Vessels: Uncertainty and Sensitivity Analysis. Journal of Loss Prevention in the Process Industries, 2021, 71, 104450, https://doi.org/10.1016/j.jlp.2021.104450

42. Vaidogas, E. R. Risk Oriented Design of Protective Highway Structures. Baltic Journal of Road and Bridge Engineering, 2007, 2(4), 155-163.

43. Vaidogas, E.R., Linkute, L. Sitting the Barrier Aimed at Protecting Roadside Property from Accidental Fires and Explosions on Road: A Pre-Optimisation Stage. Baltic Journal of Road and Bridge Engineering, 2012, 7(4), 277-287. https://doi.org/10.3846/bjrbe.2012.37

44. Troffaes, M. C. M., Walter, G., Kelly, D. A Robust Bayesian Approach to Modeling Epistemic Uncertainty in Common-Cause Failure Models. Reliability Engineering \& System Safety, 2014, 125(May), 13-21. https://doi. org/10.1016/j.ress.2013.05.022

45. Tugnoli, A., Cozzani, V., Khan, F., Amyotte, P. Missile Projection Effects. In: Reniers, G., Cozzani, V. (Eds.), Domino 
Effects in the Process Industries. Modelling, Prevention and Managing, Elsevier, Amsterdam, 2013, 116-153. https://doi.org/10.1016/B978-0-444-54323-3.00006-3

46. Walley, P. Inferences from Multinomial Data: Learning About a Bag of Marbles. Journal of the Royal Statistical Society, Series B, 1996, 58(1), 3-34. https://doi. org/10.1111/j.2517-6161.1996.tb02065.x

47. Wilson, K. J. Specification of Informative Prior Distributions for Multinomial Models Using Vine Copulas. Bayesian Analysis, 2018, 13(3), 749-766. https://doi. org/10.1214/17-BA1068
48. Yong, A. C. Y., Lam, N. T. K., Menegon, S. J., Gad, E. F. Cantilevered RC Wall Subjected to Combined Static and Impact Actions. International Journal of Impact Engineering, 2020, 143, 103596. https://oi.org/10.1016/j. ijimpeng.2020.103596

49. Zubair, M., Amjad, Q. M. N. Calculation and Updating of Common Cause Failure Unavailability by Using Alpha Factor Model. Annals of Nuclear. Energy, 2016, 90(April), 106-114. https://doi.org/10.1016/j.anucene.2015.12.004 